\title{
Tuning the Composition and Morphology of Carbon Nanotube-copper
}

\section{Interface}

Farhad Daneshvar ${ }^{\mathrm{a}}$, Tan Zhang ${ }^{\mathrm{a}}$, Atif Aziz ${ }^{\mathrm{b}}$, Hung-Jue Sue ${ }^{\mathrm{a} *}$, and Mark E. Welland ${ }^{\mathrm{b}}$

a. Polymer Technology Centre, Department of Materials Science and Engineering, Texas A\&M University, College Station, TX 77843, USA.

b. Nanoscience Centre, Department of Engineering, University of Cambridge, CB3 OFF, UK.

* Corresponding author: Hung-Jue Sue, E-mail: hjsue@tamu.edu

\begin{abstract}
Carbon nanotube (CNT)-copper nanocomposites are promising materials for light-weight highampacity conductors. The key issue in hybridization of CNT and metal is creating a strong bonding between them. This requires proper modification of the CNT surface. However, experimental works on determining the effect of interfacial functionalization on the properties of the metal/CNT system is scarce. In this paper, the effect of CNT surface modification on the morphology, interfacial interactions, and electrical properties $\mathrm{Cu} / \mathrm{CNT}$ hybrid system is investigated. For this purpose, CNTs with carboxyl, thiol, and nitrogen doped surface groups were used as substrate for growth of $\mathrm{Cu}$ particles via a facile electrochemical process. It is observed that not only the morphology but also the chemical state of the $\mathrm{Cu}$ deposits are affected by the surface functional group on CNT. Thiol group significantly enhances copper wettability towards CNT, facilitating a uniform deposition of copper, and impedes oxidation. Also, $\mathrm{Cu} / \mathrm{CNT}$ thin films fabricated via vacuum filtration that contain thiol-activated CNTs shows four and seven times higher electrical conductivity compared with systems that contain carboxyl and Ndoped surface groups, respectively.
\end{abstract}


Keyword: Carbon nanotube, surface modification, interfacial interaction, copper, electrical conductivity.

\section{Introduction}

Copper has been the conductive material of choice in electronic devices for years due to its superior electrical conductivity, abundance, and thermal resistance among others. Future technological advances, from portable electronics to space explorations, demand electronics with smaller size and lower weight, parameters that metals such as copper fail to satisfy. However, recent developments in nanotechnology have provided new materials which possess the potential to surpass copper in terms of electrical conductivity, weight, and ampacity. Among these, carbon nanotubes (CNTs) stand out on the basis of their high electrical and thermal conductivity, remarkable ampacity, low density, abundance of precursor materials, and supreme mechanical properties [1].

Individual CNTs are hollow quantum wires with extremely narrow diameters in the range of several atomic distances. The charge carriers are free to travel only along the axial directions and show ballistic electron transport at room temperature [2]. Nevertheless, research into translating these properties from nanoscale to macro scale have not been straightforward. Attempts to fabricate highly conductive macrostructures like sheets and yarns have shown some promising results, but only through complex processing and intensive and expensive chemical treatments [3-6]. A different approach, inspired by the theoretical work of Hjorstam et al. [7], is to employ the CNTs as nanofillers in copper matrix. By simple effective-medium model, it was predicted that at a 30-40 vol\% loading, metallic single-walled CNTs (SWCNTs)-copper composites could 
present room temperature electrical conductivities about twice that of annealed copper. This conclusion is of course under ideal conditions, such as uniform dispersion of CNTs and a perfect interface with the matrix. Unfortunately, experimental efforts have not been able to achieve these predicted conductivities even though a tremendous potential has already been demonstrated. For example, Subramaniam et al. [8] fabricated a Cu-CNT (45 vol\%) sheet that had room temperature conductivity comparable to copper (81\%), and ampacity and specific conductivity 100 times and $26 \%$ higher than copper, respectively.

One of the remedies to close the gap between theoretical and experimental results is to enhance the interfacial interactions between the CNT and the matrix. For polymer-based composites, this subject has been widely studied leading to development of numerous CNT surface treatment approaches with successful results [9-12]. These surface modification techniques have also been implemented in CNT-metal nanocomposites with some promising results, especially in terms of mechanical properties [13-18]. Nonetheless, research that have experimentally and systematically compared the effect of different surface groups on CNT-metal interface are scarce. These studies are necessary when significant number of research efforts are focusing on the CNT-metal and metal oxide systems in various applications such as energy storage, sensors, catalysts, and portable electronics $[1,19,20]$.

In the present work, the effect of CNT surface treatment on the morphology, structure, and electrical conductivity of the $\mathrm{Cu}-\mathrm{CNT}$ system has been investigated. Compared to other metals, $\mathrm{Cu}$ has weak interactions with CNTs [21], but this issue has been tackled in this work by utilizing a proper surface treatment and synthesis method. Three of the most common and promising functionalization groups have been chosen for the study, namely, carboxyl (two different conditions), N-doping and thiol. Functionalized CNTs are coated in nanoscale via a 
facile and versatile electrochemical method. Our findings show that not only the morphology but also the composition of the metallic deposits are affected by the CNT surface treatment. Thiol group, which is known to have strong interactions with precious metals [22], shows the best affinity towards $\mathrm{Cu}$. The thiol activated CNTs are uniformly coated with $\mathrm{Cu}$ and show electrochemical stability. Subsequently, Cu-seeded CNTs with different functionalities were used to fabricate $\mathrm{CNT} / \mathrm{Cu}$ thin films. These composite thin films are produced via vacuum filtration and a subsequent electroless deposition methods. It is observed that CNT surface groups have significant effect on the electrical conductivity of these fibers; the thiol-activated $\mathrm{CNT} / \mathrm{Cu}$ system shows the highest conductance, four and seven times higher than systems containing carboxyl and N-doped CNTs, respectively. Our results show that by proper tailoring of the CNT surface functionality, one can develop light-weight $\mathrm{Cu}$-CNT nanocomposite wires with remarkable conductivity. The present findings can be used in a wide variety of applications, such as CNT (or graphene)-inorganic hybrid materials for energy storage, sensors, and catalysts; applications in which high surface area and electrical conductivity are desired.

\section{Materials and methods}

\subsection{Materials:}

Multi-walled CNTs (MWCNT) with a purity of $95 \mathrm{wt} . \%$, diameter of $10 \mathrm{~nm}$ and length of 1-10 $\mu \mathrm{m}$ were supplied by Arkema Inc. To be concise, they will be referred to as CNTs. All other chemicals were obtained from Sigma-Aldrich and were used as received.

\subsection{Pretreatment of CNTs}

To study the effect of surface chemistry, CNTs were subjected to 4 different functionalization treatments, as described below. 
1) Mild oxidation: $200 \mathrm{mg}$ of as received-CNTs were dispersed in a mixture of sulfuric ( $75 \mathrm{~mL})$ and nitric acids $(25 \mathrm{~mL})$ and ultrasonicatated in a sonication bath for two hours at room temperature. Subsequently, $150 \mathrm{~mL}$ deionized water $\left(\mathrm{DI}-\mathrm{H}_{2} \mathrm{O}\right)$ was added and the solution was sonicated for another hour at room temperature. After oxidation, oxidized MWCNTs were washed four times with DI- $\mathrm{H}_{2} \mathrm{O}$ and collected on a polyvinylidine fluoride (PVDF) filter membrane (Millipore, Durapore, $0.45 \mu \mathrm{m}$ pore size) under vacuum. Finally, they were dispersed in DI- $\mathrm{H}_{2} \mathrm{O}$. From here on these mildly oxidized MWCNTs are going to be referred as MO-CNTs.

2) Harsh oxidation: Similar to the first surface treatment process except that before diluting the CNTs with DI- $\mathrm{H}_{2} \mathrm{O}$, they were stirred for six hours at $70{ }^{\circ} \mathrm{C}$ under reflux. They are referred to as HO-CNTs from here on.

3) Nitrogen-doping: N-doping has a low interference with unique structure of CNTs. Also, by first principle calculations, it was recently shown to effectively enhance both mechanical and electrical properties of Cu-CNTs [23]. Respectively, N-doped CNTs (N-CNTs) were provided by from Arkema Inc., and used as substrate for deposition of Copper. According to the supplier, the MWCNTs have an average diameter of $10 \mathrm{~nm}$ and average length of $5 \mu \mathrm{m}$ with 7 at $\% \mathrm{~N}$.

4) Thiol functionalization: $100 \mathrm{mg}$ MO-CNTs obtained from the mild oxidation process described above are dispersed in $200 \mathrm{~mL}$ ethanol. N, N'-Dicyclohexylcarbodiimide (DCC) was added to the solution in weight ratio of $1: 1 \mathrm{w} / \mathrm{w}$ compared with CNTs. This mixture was then sonicated for $30 \mathrm{~min}$ and then stirred at room temperature for one hour. Next, $150 \mathrm{mg}$ cysteamine was added in a weight ratio of 3:2 compared with MWCNTs and the mixture was stirred at room temperature for 24 hours. Thiol activated MWCNTs (SH-CNTs) were washed at least four times using PVDF filter membrane (Millipore, Durapore, $0.45 \mu \mathrm{m}$ pore size) under vacuum. 


\subsection{Preparation of $\mathrm{Cu} / \mathrm{CNTs}$}

CNTs with different surface groups (f-CNTs) were similarly coated with $\mathrm{Cu}$ using electroless deposition technique as described in [19]. Concisely, $50 \mathrm{mg}$ of f-CNTs were dispersed in $100 \mathrm{~mL}$ aqueous solution of $\mathrm{SnCl}_{2}(1 \mathrm{~g})$ and $\mathrm{HCl}(0.25 \mathrm{~mL})$. The mixture was sonicated for $30 \mathrm{~min}$ at room temperature. Next, Sn-sensitized CNTs were activated using $\mathrm{PdCl}_{2}(0.01 \mathrm{~g})$ and $\mathrm{HCl}(0.25$ $\mathrm{mL}$ ) $50 \mathrm{~mL}$ aqueous solution by ultrasonication for $30 \mathrm{~min}$ at room temperature followed by rinsing in DI- $\mathrm{H}_{2} \mathrm{O}$ using centrifuge. This washing step should be done carefully to remove all the excessive or unattached Pd and Sn ions from the solution. Finally, the activated CNTs were introduced to a $100 \mathrm{~mL} \mathrm{Cu}$ electroless deposition bath. The composition and conditions of the plating bath are given in Table 1 .

Table 1. The composition and conditions of the copper electroless bath deposition.

\begin{tabular}{ll}
\hline Chemical composition & Quantity \\
\hline $\mathrm{CuSO}_{4} .5 \mathrm{H}_{2} \mathrm{O}$ & $6.2 \mathrm{~g} / \mathrm{l}$ \\
2Na-EDTA & $40 \mathrm{~g} / 1$ \\
$\mathrm{CHOH}\left(37 \mathrm{vol} \%\right.$ in $\left.\mathrm{H}_{2} \mathrm{O}\right)$ & $20 \mathrm{ml} / 1$ \\
Temperature & $60^{\circ} \mathrm{C}$ \\
$\mathrm{pH}(\mathrm{NaOH})$ & 13 \\
\hline
\end{tabular}

\subsection{Fabrication of CNT-Cu film for electrical conductivity measurements}

Vacuum filtration was utilized for fabrication of CNT-Cu films similar to [24]. Firstly, $50 \mathrm{mg} \mathrm{f}$ CNTs were seeded by copper using similar electroless deposition process explained in section 2.3. The only difference is that the concentration of all the metallic salts and 2Na-EDTA were cut in half to minimize the free particle formation and to promote copper nucleation. Free metallic particles and impurities were removed by filtering and centrifuge at $2000 \mathrm{rpm}$ for $5 \mathrm{~min}$. To fabricate the thin films, the aqueous solutions containing f-CNT/Cu hybrid systems were vacuum-filtered using polytetrafluoroethylene (PTFE) membrane filters with pore size of $450 \mathrm{~nm}$ 
(Millipore). The f-CNT/Cu were dried using a vacuum oven at $50{ }^{\circ} \mathrm{C}$ for $12 \mathrm{~h}$. To ensure all the hybrid systems have similar amount of copper, dried copper seeded f-CNT films were electroless plated in $150 \mathrm{~mL}$ of copper bath with composition presented in Table 1 for 10 to $12 \mathrm{~min}$. The copper coated films were dried using a vacuum oven at $50^{\circ} \mathrm{C}$ overnight. The thin films with different CNT functional groups have the same weight after this process. Finally, the $\mathrm{f}-\mathrm{CNT} / \mathrm{Cu}$ films were heated at $250^{\circ} \mathrm{C}$ for two hours in controlled flow of nitrogen and immediately followed by one hour in nitrogen-hydrogen (5 vol\%) gas under $1 \mathrm{kPa}$ pressure using dynamic mechanical analyzer (DMA) (ARES 300-G2, TA instruments). For comparison, the same process was used to fabricate thin film of pristine MWCNTs (P-CNTs).

\subsection{Characterization}

The structural properties and the morphologies of the synthesized $\mathrm{Cu} / \mathrm{MWCNT}$ hybrid systems were examined using transmission electron microscopy (TEM) (FEI Teccai G2 S-Twin, Philips), X-Ray diffraction (XRD) (Bruker D8 Advance ECO) with CuKa incident radiation $(\lambda=0.1506$ $\mathrm{nm}$ ), and X-ray photoelectron spectroscopy (XPS) (Omicron DAR 40 dual Mg/Al x-ray source). XPS plots were deconvoluted based on Gaussian-Lorentzian peaks and Shirley background subtraction. Prior to XRD and XPS experiments the samples were filtered and centrifuged to remove all the free $\mathrm{Cu}$ particles formed in the solution. FEI Tecnai Osiris S/TEM working at 200 $\mathrm{keV}$ was used to obtain STEM and EDX. The EDX detectors were FEI Super-X systems employing 4 Bruker silicon drift detectors for high collection efficiency ( $>0.9$ sr solid angle) and high count rates (>250 kcps).

To investigate the effect of surface treatment on electrical conductivity of the hybrid systems, thin films of $\mathrm{Cu} / \mathrm{MWCNT}$ were made using vacuum filtering approach. Four-point-probe conductivity measurements were carried out on free-standing $\mathrm{Cu} / \mathrm{MWCNT}$ films with a four- 
point-probe (Signatone, SP4-40045TBY) on a resistive stand (Signatone, Model 302). The conductivity was calculated by using the formula:

$\sigma=\frac{I \cdot \ln 2}{\pi \cdot V \cdot t \cdot k}$

where $\sigma$ is the electrical conductivity $(\mathrm{S} / \mathrm{m}), \mathrm{I}(\mathrm{A})$ is the current going through the outer two probes, $\mathrm{V}(\mathrm{V})$ is the voltage drop measured across the two inner probes, $\mathrm{t}$ is the thickness of the film (m) (which was obtained by SEM), and $\mathrm{k}$ is a correction factor. Three to four samples were analyzed for each functionalization method and 10 to 15 measurements were carried out on each sample. The average and standard deviation are reported.

\section{Results and discussion}

\subsection{Surface treatment of CNTs}

XPS was used to investigate the surface and interfacial chemistry of functionalized CNTs. The results show that all the samples contain $\mathrm{C}$ and $\mathrm{O}$. In addition, $\mathrm{N} 1 \mathrm{~s}$ peak at $399.3 \mathrm{eV}$ and S 2p peak at $169.3 \mathrm{eV}$ are detected for N-CNT and SH-CNT, respectively. The C 1s peak for all the samples is situated almost at the same binding energy of $284.35 \mathrm{eV}$, which will be further analyzed in detail. It should be added that the $\mathrm{O} 1 \mathrm{~s}$ peak binding energy depends on the surface modification technique and varies from $531.5 \mathrm{eV}$ for $\mathrm{SH}-\mathrm{CNT}$ to $532.5 \mathrm{eV}$ for MO-CNT (Figure S1).

The high resolution XPS data of C 1s peaks for f-CNTs and their deconvoluted peaks are represented in Figures 1a and 1b. As can be observed, MO-CNT sample is mainly consisted of $\mathrm{C}=\mathrm{C}(284.3 \mathrm{eV})$ and $\mathrm{C}-\mathrm{C}(285.5 \mathrm{eV})$ structures. The strong $\mathrm{C} 1 \mathrm{~s}$ peak corresponding to $\mathrm{sp}^{2}$ carbon, which is more or less common for other samples as well, shows that the conjugated bonds in CNT lattice are preserved after the surface treatments. Also, C-O-C (or C-O) peak at 
$286.6 \mathrm{eV}, \mathrm{O}-\mathrm{C}=\mathrm{O}$ peak (or $\mathrm{C}=\mathrm{O}$ ) at $288.3 \mathrm{eV}, \mathrm{C}-\mathrm{OOH}$ peak at $289.5 \mathrm{eV}$, and broad $\pi-\pi$ peak at $291.5 \mathrm{eV}$ are resolved [25]. With increasing the intensity of the acid treatment, the $\mathrm{C} 1$ peak becomes more asymmetric due to emergence of the shoulder at higher binding energies. The resolved peaks show that the relative intensity of $\mathrm{C}=\mathrm{C}$ peak $\left(\mathrm{sp}^{2}\right)$ slightly decreases while the $\mathrm{C}$ $\mathrm{C}$ and oxygen containing species become stronger suggesting that the CNT structure is more distorted and more defects are introduced to the HO-CNT surface. The carboxyl group content in each sample is estimated based on the area beneath the resolved peaks. The result shows that carboxyl group content in MO-CNT and HO-CNT samples are around 7.5 at $\%$ and 12 at\%, respectively. Moreover, based on the deconvolution of O 1s, the HO-CNT sample contained contains relatively higher $\mathrm{C}=\mathrm{O}$ bonds concentration (Figure $\mathrm{S} 1$ ).

Compared to carboxyl functionalized samples, the full width at half maximum (FWHM) is higher for N-CNT and SH-CNT samples even though the oxygen containing groups have almost the same intensity. This is attributed to formation of a new peak at $286.0 \mathrm{eV}$ which is ascribed to C-N for N-CNT and C-S or C-N for SH-CNT samples. Finally, compared with MO-CNT and HO-CNT samples, the intensity of oxygen containing groups decrease in the N-CNT and SHCNT samples, which indicates that the oxygen functionalities reduction occurs by $\mathrm{N}$-doping and thiol grafting. The nature of these changes can be revealed by analyzing the high resolution XPS of $\mathrm{N} 1 \mathrm{~s}$ and $\mathrm{S} 2 \mathrm{p}$ respectively.

High resolution XPS shows that the N 1s core level spectra for N-doped CNTs (Figure 1c) can be deconvoluted in two peaks related to pyrodinic $\mathrm{N}$ at $298.7 \mathrm{eV}$ and graphitic $\mathrm{N}$ at $400.7 \mathrm{eV}$ [26]. As illustrated in Figure 1e, the former represents the substitution of an $\mathrm{N}$ atom with a $\mathrm{C}$ atom adjacent to a defect while the latter represents substitution of $\mathrm{N}$ atom with a $\mathrm{C}$ atom in a perfect benzene ring [26]. Moreover, the atomic ratio of these two structures are calculated by 
integrating the fitting curve area of $\mathrm{N} 1$ s peak, showing that doped $\mathrm{N}$ is almost 70 at $\%$ in pyrodinic form and the rest is in graphitic form.

Figure 1d displays the S 2p spectra corresponding to the SH-CNT showing that the thiol groups are successfully formed on the CNT's surface. S 2p has two main peaks with one situated at 168 $\mathrm{eV}$ which is attributed to sulfates and one around $164 \mathrm{eV}$ which is a result of cysteine and thiol formation. The latter is the peak of interest here. Cysteine peak fitting results revealed that it can be deconvoluted to three components corresponding to C-S $2 \mathrm{p}_{1 / 2}$ at $163.3 \mathrm{eV}, \mathrm{C}-\mathrm{S} 2 \mathrm{p}_{3 / 2}$ at 164.5 $\mathrm{eV}$ and a sharp peak at $163.9 \mathrm{eV}$ which is assigned to $\mathrm{SH}$ tail of the thiol group [27]. These results suggest that thiol groups are formed by the amidation reaction between the amine groups of cysteine and the carboxylic groups of CNTs [27]. The surface composition of the CNTs was also determined using XPS, which indicated a 3.2 at $\%$ of S. 

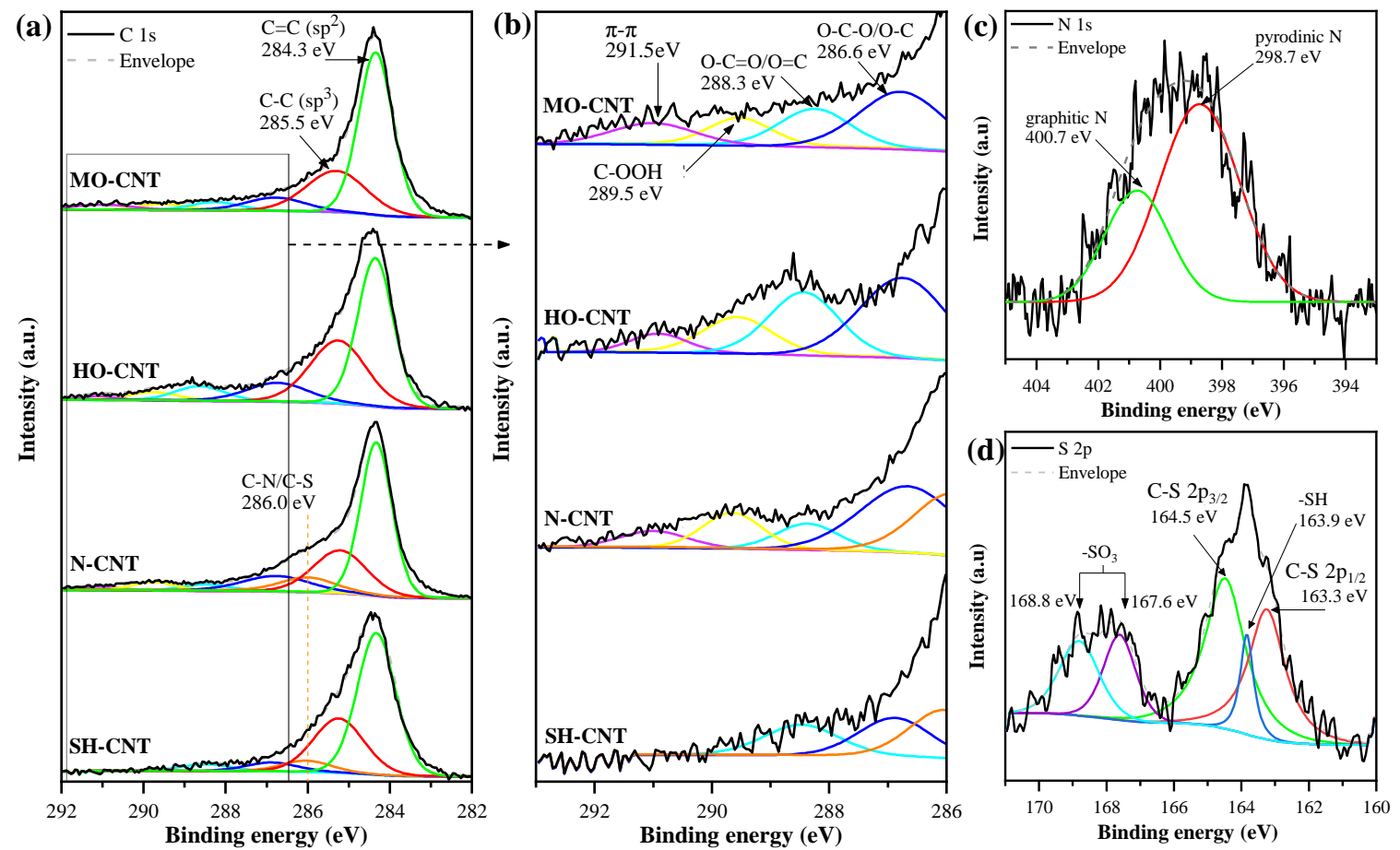

(e)
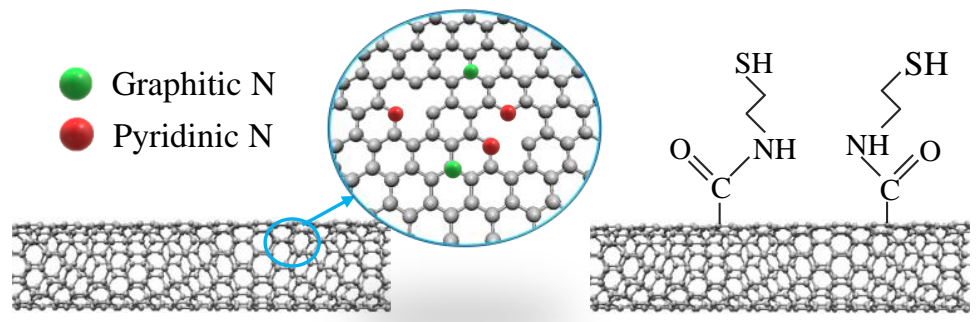

Carboxyl-activated CNT

$\mathrm{N}$-doped CNT

Thiol-activated CNT

Figure 1. High resolution XPS spectra of (a) C1s, (b) C1s between 286-293 eV of the CNTs with different surface groups, (c) N 1s peak of N-CNT system, and (d) S 2p of SH-CNT. The dominant surface functional group for each type of surface treatment is schematically presented in (e). It should be noted that both $\mathrm{MO}$ and $\mathrm{HO}$ treatments create similar surface functional groups but in the latter these surface groups will be more abundant.

\subsection{Morphology and composition of $\mathrm{Cu} / \mathrm{CNT}$ systems}

TEM analyses of the hybrid systems show that the functional group on the CNT surface can significantly affect the morphology and density of the copper deposits. Figure 2 shows a representative set of TEM images for $\mathrm{Cu} / \mathrm{CNT}$ hybrid systems for four different functional groups. In $\mathrm{Cu} / \mathrm{MO}-\mathrm{CNT}$ system, $\mathrm{Cu}$ deposits randomly and scarcely cover the MO-CNTs surface. While some of the CNTs have no or only a small amounts of $\mathrm{Cu}$, some large deposits can be observed specifically at the tube ends showing that seeding of Pd and subsequently deposition of $\mathrm{Cu}$ do not occur uniformly on all the CNTs. 
This defect-dependence deposition has been previously reported in electroless deposition of $\mathrm{Cu}$ on graphene [28]. CNT ends and defects are proper sites for deposition of $\mathrm{Cu}$. Due to the anisotropy of CNT system, carbon atoms at the tips are more reactive, can form strong covalent bonds and show stronger interactions with metals [29]. Moreover, CNT sidewalls are resistant to acid treatment so oxidation usually initiates at and mostly affects nanotube tips and defects [30]. As a result, oxidation in the mild acid treatment does not occur uniformly and the distance between proper nucleation centers may be too far from each other to form a continuous $\mathrm{Cu}$ layer. The scattered deposits on the sidewalls have smaller size and are readily oxidized which impedes the autocatalytic growth of $\mathrm{Cu}$. On the other hand, the already defective tube ends are mostly affected by acids and carboxyl groups are densely generated at these locations. This leads to formation of scattered $\mathrm{Cu}$ deposits on the MO-CNT sidewalls and big $\mathrm{Cu}$ aggregates at the tips. It should be noted that free $\mathrm{Cu}$ particles were observed in this sample (not shown here) possibly due to detachment of Pd particles from the surface during rinsing or detachment of $\mathrm{Cu}$ particles during sonication prior to TEM sample preparation. Due to high curvature, the surface energy of CNTs is high. Therefore, if a strong interaction between the CNT and the metallic particles does not exist, they will detach from the CNT side-wall. Therefore, MO-CNT fails to provide a proper substrate for nucleation and growth of $\mathrm{Cu}$ layer. 

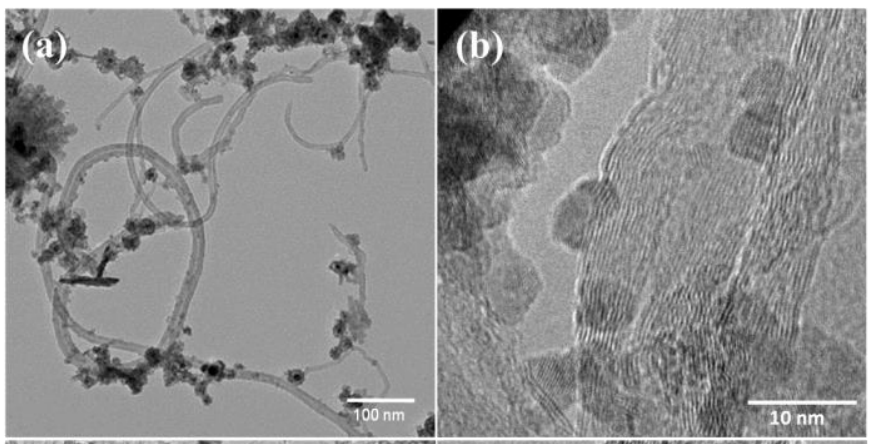

(i)
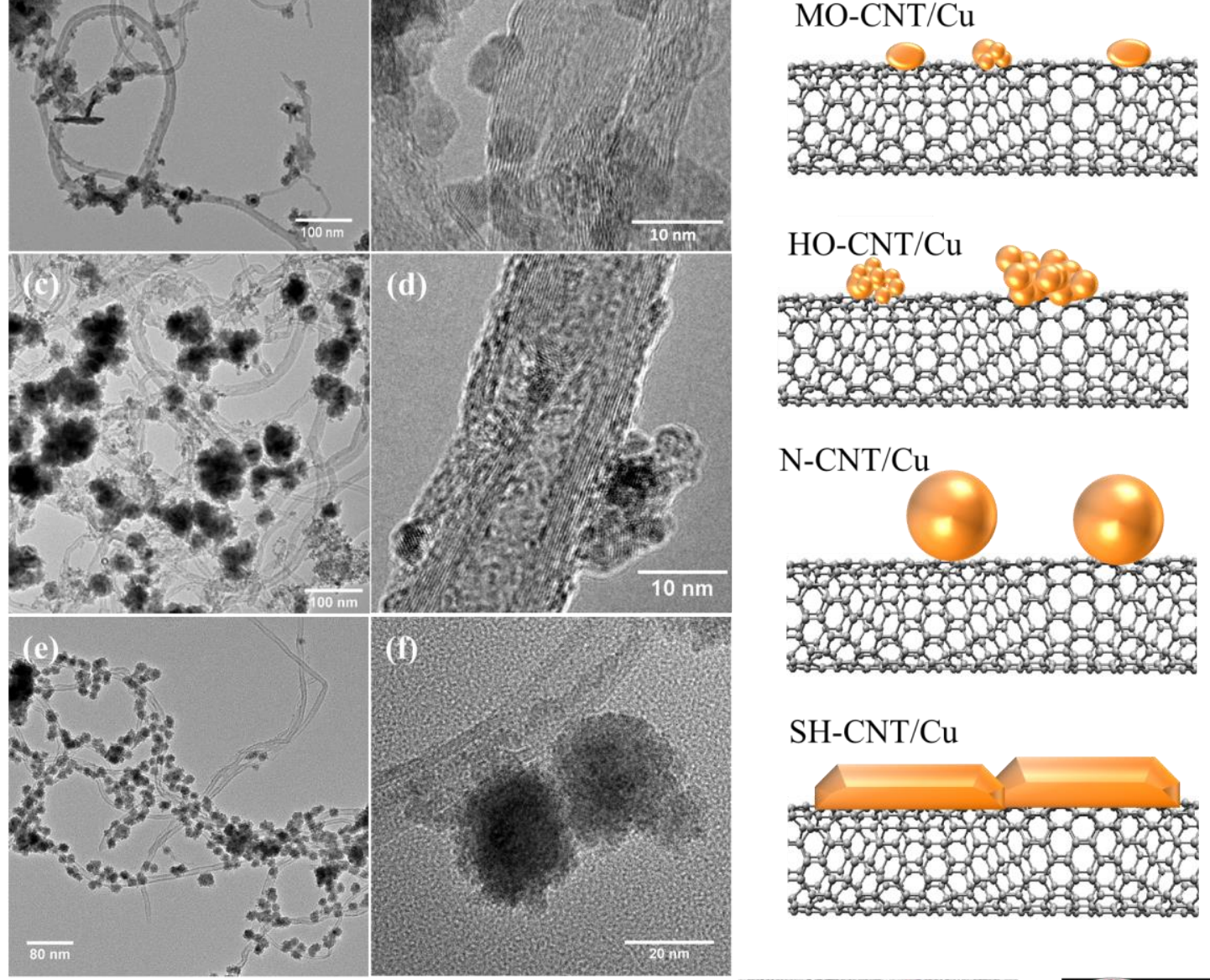

\section{$\mathrm{SH}-\mathrm{CNT} / \mathrm{Cu}$}
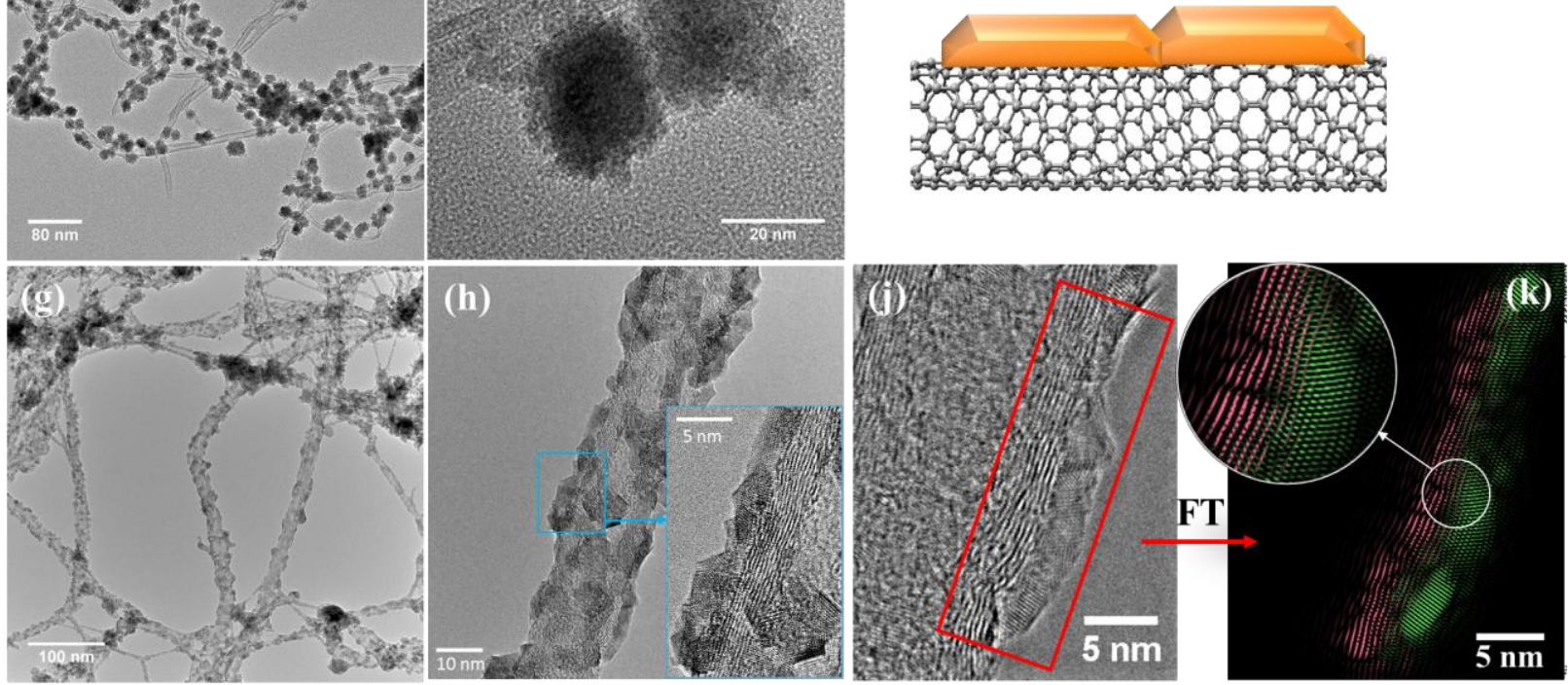

Figure 2. Morphology of the Cu deposits on the sidewalls of CNTs with different functional groups. Scattered $\mathrm{Cu}$ deposits with diameter $\sim 4 \mathrm{~nm}$ formed on the surface of MO-CNTs (a-b). Big aggregates of $\mathrm{Cu}(\sim 100 \mathrm{~nm})$ are observed at the HO-CNT tube ends along with scattered deposits on the sidewalls (c-d). When the CNTs are doped with nitrogen $\mathrm{Cu}$ aggregation at the tube ends decreases and sphere shape deposits $(\sim 20 \mathrm{~nm})$ are formed on the sidewalls while some of the N-CNTs are not coated at all (e-f). SH-CNT surface is wetted by $\mathrm{Cu}$ and all the SHCNTs are uniformly coated with a thin layer of $\mathrm{Cu}(\sim 4 \mathrm{~nm})$ and negligible $\mathrm{Cu}$ aggregation is observed at tube ends (g-h). The effect of CNT's surface group on the morphology of the Cu deposits is schematically represented in (i). High resolution TEM of SH-CNT/Cu at the interface (j) and the corresponding processed image based on Fourier transformation (FT) shows how $\mathrm{Cu}$ deposits (green) are integrated in CNT (purple) (k). 
By increasing the intensity of the acid treatment, more carboxyl groups are expected to from on the surface of CNTs which provides abundant proper locations for nucleation of $\mathrm{Pd}$ and $\mathrm{Cu}$, and leads to a denser $\mathrm{Cu}$ coverage. However, this phenomenon is not observed in our work. By increasing the surface oxidation intensity, the density of the $\mathrm{Cu}$ deposits on the CNT sidewalls shows a slight enhancement while the size of the deposits shows a more vivid increase; specifically large aggregates are formed at the tube ends. In addition to the deposit size, acid treatment intensity also affects the morphology of the deposits. As observed in high resolution TEM image of MO-CNT/Cu system (Figure 2b), the Cu deposits have a regular oval shape with diameter around $4 \mathrm{~nm}$. However, in $\mathrm{HO}-\mathrm{CNT} / \mathrm{Cu}$ sample, the $\mathrm{Cu}$ deposits have irregular shapes and are generally larger. Based on shape irregularity and different fringe directions, it is speculated that they are formed by growth and aggregation of a few $\mathrm{Cu}$ nuclei. It should be noted that based on the zeta potential measurements (Figure S2), more aggressive acid treatment results in a more negative surface charge in HO-CNTs. By considering the XPS results, it can be deduced that larger and more abundant defect sites are formed on the HO-CNT surface. However, these sites are not uniformly distributed on the CNT surface and large $\mathrm{Cu}$ aggregates (that can reach to $100 \mathrm{~nm}$ in size) are formed at the tips of some of the CNTs (Figure S3). While these aggregates were also observed on MO-CNT, their size rarely exceeded $40 \mathrm{~nm}$. Strong electrostatic forces at these locations attracts more $\mathrm{Pd}^{+2}$ and $\mathrm{Cu}^{2+}$ ions. Therefore, large spheres at the HO-CNT tube ends are the result of formation of functional groups, and subsequently larger catalytic particles that effectively initiates $\mathrm{Cu}$ deposition.

The deposits on the surface of the N-CNTs have a more uniform shape and morphology. TEM results show that most of the N-CNTs sphere metallic deposit with diameter around $20 \mathrm{~nm}$ (Figures 2e and 2f). Similar to carboxyl group, N-doped CNTs contains atoms with lone electron 
pair which theoretically can bind with metal ions through sharing an electron pair [31]. Recently, by theoretical and experimental studies, Milowska et al. [23] showed that N-doping of CNTs would enhance the adhesion of sputtered $\mathrm{Cu}$ to CNTs and results in a composite with improved mechanical and electrical properties. However, N-doped CNTs are hydrophobic and show poor dispersion in water compared to MO-CNTs and HO-CNTs. This can impede interactions between $\mathrm{Pd}^{+2}$ and $\mathrm{Cu}^{2+}$ ions with $\mathrm{N}$-doped CNTs in aqueous solutions. In addition, $\mathrm{N}$-doped CNTs are protonated in water which shifts the $\mathrm{pH}$ at point of zero charge $\left(\mathrm{pH}_{\mathrm{zpc}}\right)$ to higher values compared with MO-CNTs and HO-CNTs (Figure S2). This will cause a repulsive electrostatic force between divalent metal ions, such as $\mathrm{Cu}^{2+}$ and N-CNTs, and reduces the $\mathrm{Cu}$ adsorption on $\mathrm{N}-\mathrm{CNTs}$ ' surface. On the other hand, the $\mathrm{N}$ atoms are able to share their electrons with the $\mathrm{Pd}^{+2}$ and/or $\mathrm{Cu}^{2+}$ ions. The combination of repulsive forces between the hydrophobic inert CNT surface and the ions, and the attraction of metallic ions to the pyrodinic $\mathrm{N}$ can lead to the formation of spherical $\mathrm{Cu}$ structure with minimal contact to the CNT surface, as observed in Figures $2 \mathrm{e}$ and $2 \mathrm{f}$.

The morphology of the SH-CNT/Cu hybrid system is represented in Figures $2 \mathrm{~g}, 2 \mathrm{~h}$ and $2 \mathrm{j}$. The crystalline structure of SH-CNTs indicates that the CNT structure is preserved after surface treatment. In addition, the SH-CNTs are more uniformly coated and almost all of the CNTs are covered by $\mathrm{Cu}$ deposits. It is well-know that thiol has strong affinity for some metals and can bond with them through its sulfur group [32-34]. Moreover, as previous studies have shown when CNTs are thiol activated, the noble metal particles are not agglomerated but uniformly attached to CNT sidewall surface $[32,33]$. As a result, it can be deduced that Pd has been seeded uniformly on SH-CNT surface. Cu nucleation is initiated from these seeds and then grow and form $\mathrm{Cu}$ clusters, forming a uniform coating on SH-CNT surface. By investigating at the $\mathrm{Cu}$ 
deposits morphology at higher magnifications, another interesting difference against other treatment conditions becomes apparent. Unlike the MO-CNT/Cu and $\mathrm{HO}-\mathrm{CNT} / \mathrm{Cu}$ samples, the $\mathrm{Cu}$ particles on $\mathrm{SH}-\mathrm{CNT}$ are not spherical but have elongated trapezium shape, showing a better wetting toward the SH-CNT surface, suggesting that there is a stronger interaction between $\mathrm{Cu}$ and $\mathrm{SH}-\mathrm{CNT}$ (Figure 2j). As a result, CNT surface functionality strongly influences not only the $\mathrm{Cu}$ coverage but also their shape.

STEM images and nanoscale elemental maps of the electroless deposited CNTs are presented in Figure 3. For each sample several locations were investigated. In all cases, $\mathrm{Cu}$ was the main constituent of the deposits along with oxygen and in tin. Also, in almost all the cases, Pd was not detected showing its content is below the detection limits of the equipment. The only exception was the $\mathrm{HO}-\mathrm{CNT} / \mathrm{Cu}$ sample in which Pd was detected in specific areas (Figure S4). As it was discussed before, this sample contains relatively large $\mathrm{Cu}$ particles which can be formed due to accumulation of catalytic seeds. Pd was only detected around the large aggregates showing that indeed they are formed due to densely packed catalyst particles at HO-CNT defect sites. The corresponding EDX plots are provided in the supplementary (Figure S5).

XPS was used to analyze the surface chemistry of functionalized CNTs and hybrid systems. After $\mathrm{Cu}$ deposition, all the hybrid systems contain $\mathrm{Cu}, \mathrm{O}, \mathrm{C}$, and to a lesser extent $\mathrm{Sn}$ and $\mathrm{Pd}$ (Figure 4). Peaks at binding energies of 335, 486.6 and $932.5 \mathrm{eV}$ are associated with $\mathrm{Pd}$ $3 \mathrm{~d}, \mathrm{Sn} 3 \mathrm{~d}$ and $\mathrm{Cu} 2 \mathrm{p}$ respectively. $\mathrm{C} 1 \mathrm{~s}$ and $\mathrm{O} 1 \mathrm{~s}$ peaks are located at 284.5 and $530 \mathrm{eV}$, respectively. Except for $\mathrm{Cu} 2 \mathrm{p}$, the rest of the metallic peaks have similarly symmetric shape independent of the CNT surface functional groups. Hence, they will not be discussed further. More details about Pd 3d and Sn 3d peaks were presented in our previous work [19]. 


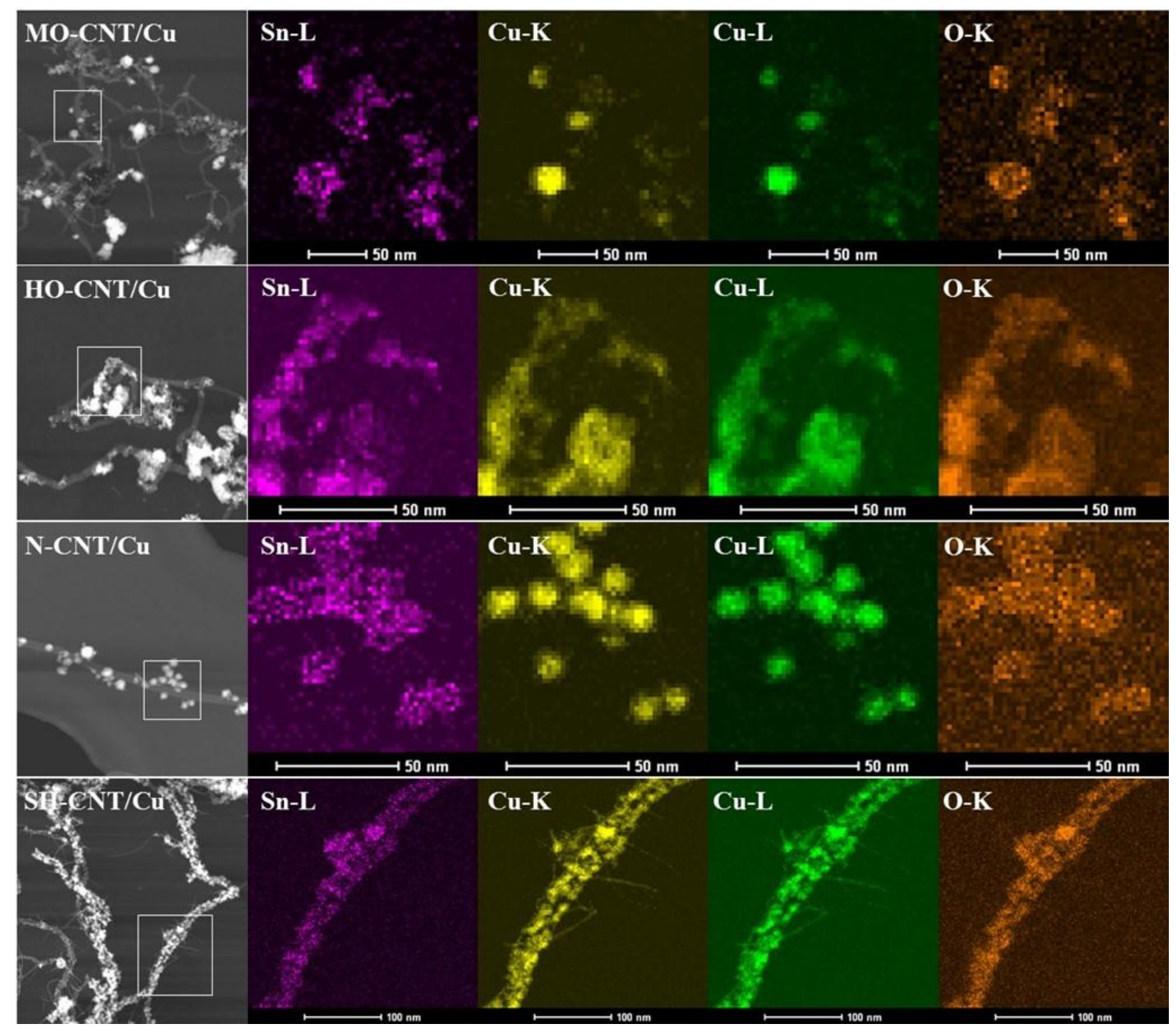

Figure 3. STEM and EDS maps of hybrid systems. $\mathrm{Cu}, \mathrm{O}$ and $\mathrm{Sn}$ are the main constituents of the coatings. No Pd was detected. Large clusters of $\mathrm{Cu}$ can be observed in MO-CNT/Cu and HO-CNT/Cu systems specifically at the $\mathrm{CNT}$ ends. In N-CNT/Cu system uniform $\mathrm{Cu}$ spheres are formed on the CNT surface. Thiol-activation will result in a uniform coating of $\mathrm{Cu}$ on the surface SH-CNTs. It seems a thin layer of tin is still present on the surface of the fCNTs.

Figure 4a shows the normalized high resolution XPS spectra of $\mathrm{Cu} 2 \mathrm{p}$ of $\mathrm{CNT} / \mathrm{Cu}$ systems. Two characteristic peaks of $\mathrm{Cu} 2 \mathrm{p}$ can be observed for all the systems. One is the $\mathrm{Cu} 2 \mathrm{p}_{3 / 2}$ at around $932.5 \mathrm{eV}$ and the other is located at binding energy of $951.6 \mathrm{eV}$, which represents the $\mathrm{Cu} 2 \mathrm{p}_{1 / 2}$ component. $\mathrm{Cu}^{2+}$, and specifically $\mathrm{Cu}^{+}$and metallic $\mathrm{Cu}$ main peaks are located very closely to each other. However, $\mathrm{CuO}$ presence can be identified by the strong satellite peaks at 961.8 and $941.6 \mathrm{eV}$, which can be attributed to metallic $\mathrm{Cu}$ or $\mathrm{Cu}_{2} \mathrm{O}$ [35-38]. Compared with other systems, 
$\mathrm{MO}-\mathrm{CNT} / \mathrm{Cu}$ system contains significantly stronger satellite peaks which indicates that a significant amount of $\mathrm{CuO}$ is present in this sample. Accordingly, less pronounced satellite peaks are detected for $\mathrm{HO}-\mathrm{CNT} / \mathrm{Cu}$ and $\mathrm{SH}-\mathrm{CNT} / \mathrm{Cu}$ systems while they are not observed for N$\mathrm{CNT/Cu}$ sample. Although during electroless deposition process, reducing and complexing agents were used, due to the small size of the particles, detection of metallic oxide is not surprising. It has previously been shown that CNTs inclusion into metallic systems can result in structure refinement $[1,13,14]$. Consequently, high surface area of these nanoparticles makes them more susceptible to oxidation [39-41]. In addition to the composition, oxidation also affects the coating morphology. $\mathrm{CuO}$ nanoparticles are inert and can hinder autocatalytic reduction of $\mathrm{Cu}$. Therefore, it is rational to assume that $\mathrm{Cu}$ on MO-CNT system is readily oxidized after reduction by formaldehyde which hampers the autocatalytic reduction and leads to discrete and small deposits of $\mathrm{CuO}$ on CNTs. On the other hand, at CNT ends where functionalization is more intense, larger $\mathrm{Cu}$ deposits are formed. In other words, in these areas, stronger interaction between the CNT and metallic particles exist. Hence, bigger and more stable catalytic sites are formed which are more resistant to oxidation. As a result, nucleation and growth becomes quicker at the CNT tube ends or other defect sites on the surface [1, 42].

The role of CNT surface chemistry on the morphology of the deposits can also be explained based on thin film growth mechanisms. During electroless deposition, $\mathrm{Cu}$ ions have some degree of mobility on the surface of CNTs. These ions travel on the surface until they are trapped at either the surface defects to form a new nucleus, or meet and join an existing $\mathrm{Cu}$ island. The coverage or uniformity of the coating is directly proportional to the diffusion rate of $\mathrm{Cu}$ ions on CNT surface. In other words, a more uniform coating can be achieved if the mobility of $\mathrm{Cu}$ ions on CNT surface is enhanced. Respectively, it is postulated that in N-CNT system, the $\mathrm{Cu}$ 
mobility on the surface of CNT is hampered. As a result, lateral growth does not occur. Also, due to poor wettability of $\mathrm{N}-\mathrm{CNT}$ surface, the $\mathrm{Cu}$ nucleus formed at the nitrogen defect grows spherically to minimize its contact and interface energy with the CNT. On the other hand, for the SH-CNT sample, the surface diffusion of ions is facilitated which leads to lateral growth of $\mathrm{Cu}$ deposits in a Volmer-Weber mode. This lateral growth continues until $\mathrm{Cu}$ islands meet each other and coalesce [43].
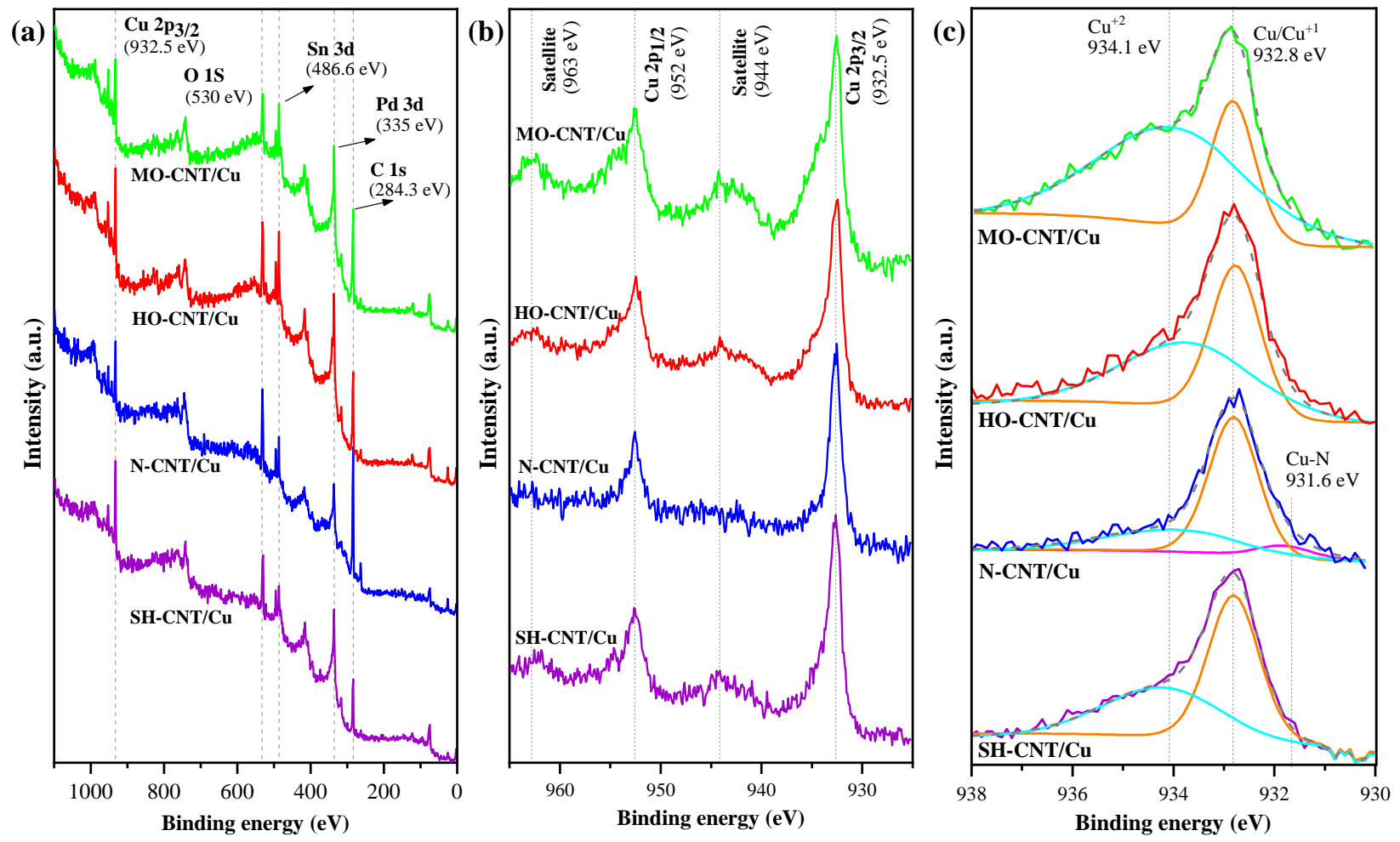

Figure 4. Wide scan spectra of hybrid systems showing that $\mathrm{Cu}, \mathrm{O}, \mathrm{Pd}$ and $\mathrm{Sn}$ are present in the coating (a). High resolution $\mathrm{Cu} 2 \mathrm{p}$ can be used to determine the state of the copper (b). Deconvoluted $\mathrm{Cu} 2 \mathrm{p}_{3 / 2}$ peaks shows that the $\mathrm{MO}-\mathrm{CNT} / \mathrm{Cu}$ system has the highest amount of $\mathrm{CuO}$ while $\mathrm{N}-\mathrm{CNT} / \mathrm{Cu}$ system has the lowest amount of $\mathrm{CuO}(\mathrm{c})$.

Compared to MO-CNT sample, weaker $\mathrm{Cu}$ satellite peaks are detected for $\mathrm{HO}-\mathrm{CNT} / \mathrm{Cu}$ and $\mathrm{SH}-$ $\mathrm{CNT} / \mathrm{Cu}$ samples showing that the $\mathrm{CuO}$ formation is limited. For more details, the $\mathrm{Cu} 2 \mathrm{p}_{3 / 2}$ peak at $932.5 \mathrm{eV}$ was resolved and the result is presented in Figure 4b. By resolving this peak, two deconvoluted peaks appear, one at binding energy of around $932.4 \mathrm{eV}$, which is attributed to $\mathrm{Cu}^{+}$ 
(i.e., $\mathrm{Cu}_{2} \mathrm{O}$ ), and the other at slightly higher binding energies which is attributed to $\mathrm{Cu}^{2+}$ (i.e., $\mathrm{CuO}$ ). It should be noted that it has been reported that formation of $\mathrm{Cu}_{2} \mathrm{O}$ thin layer on the surface is inevitable $[44,45]$. It should be noted that since metallic $\mathrm{Cu}$ peak is located almost at the same binding energy as that of $\mathrm{Cu}^{+}$, it is assumed that the peak at lower binding energies is the result of both $\mathrm{Cu}$ and $\mathrm{Cu}^{+}$components.

The molar ratio of $\mathrm{Cu} / \mathrm{Cu}_{2} \mathrm{O}$ and $\mathrm{CuO}$ are calculated based on the integrated peak area of their deconvoluted $\mathrm{Cu} 2 \mathrm{p}_{3 / 2}$ peaks [35]. Accordingly, there is 51 at $\%, 23.8$ at $\%$ and 15.8 at $\% \mathrm{CuO}$ in MO-CNT/Cu, HO-CNT/Cu, SH-CNT/Cu systems, respectively. Consequently, compared with other samples, the $\mathrm{Cu}$ in $\mathrm{MO}-\mathrm{CNT} / \mathrm{Cu}$ tends to be in higher oxidation states and more $\mathrm{CuO}$ is formed. This is attributed to the discrete deposits of finer particle size, which facilitates reaction with electrophilic neighboring protons $\left(\mathrm{H}^{+}\right)$and oxidation [46]. On the other hand, in thiol treated and harshly oxidized samples, not only particle size is larger but also proper nucleation sites are more abundant and closer to each other, which leads to formation of a continuous $\mathrm{Cu}$ layer. This will reduce the surface area and preserves the deposits from oxidation. Note that based on the previous studies, we speculate that thiol may also be able to stabilize the $\mathrm{Cu}$ deposits. Thiol has strong interactions with metallic particles due to free electron pair which forms donor centers. These donor centers are readily polarized and can form strong interaction with $\mathrm{Cu}$ ions [47]. Rigo et al. [48] studied the interactions between $\mathrm{Cu}$ and thiol using electron spin resonance (ESR), nuclear magnetic resonance (NMR). They found that thiol groups such as cysteine are able to reduce $\mathrm{Cu}^{+2}$ to $\mathrm{Cu}^{+}$probably via free radical mechanism. In addition, it has been reported that thiol has anti-oxidation effect on $\mathrm{Cu}$ nanoparticles [49] and can protect $\mathrm{Au}$ nanoparticle against chemical attacks [50]. All of these previous reports point out that thiol have 
strong interaction with metallic nanoparticles and is able to stabilize and protect them against oxidation.

In the $\mathrm{N}-\mathrm{CNT} / \mathrm{Cu}$ system, no satellite peak is observed, suggesting there is negligible amount of $\mathrm{CuO}$ in this hybrid system. As observed in Figure 2e and 2f, large spherical $\mathrm{Cu}$ particles are formed on N-CNTs with comparatively high volume to surface ratio. A thin oxide layer is formed on the surface of the particle, protecting $\mathrm{Cu}$ at the core of the sphere from oxidation. As a result, they are mostly composed of $\mathrm{Cu}$ or $\mathrm{Cu}^{+}$.

The peak shift concept in XPS can be considered to obtain a better understanding of the interactions between the $\mathrm{Cu}$ and functionalized CNTs. If there is a $\mathrm{Cu}$ peak shift towards higher binding energies, it means electron clouds are migrated to the atoms with higher electronegativities, i.e., O, C, N, or S. Also, the magnitude of the shift indicates stronger interaction $[46,51]$. For example, in $\mathrm{CuO}$, the $\mathrm{Cu}$ peak will shift to a higher binding energy because it shares some electrons with oxygen or when $\mathrm{Cu}$ bonds with sulfur. Consequently, in N$\mathrm{CNT} / \mathrm{Cu}$ sample, the $\mathrm{Cu} 2 \mathrm{p}_{3 / 2}$ peak resides at $933.1 \mathrm{eV}$, which is very close to the $\mathrm{Cu}$ binding energy reported in the literature $(933 \mathrm{eV})$ [35-38], suggesting that the $\mathrm{Cu}$ particles in this sample do not have much interaction with the N-CNT or oxygen. Moreover, the O 1s resolved peaks show that $\mathrm{Cu}$ deposition does not have any effect on the relative intensity of the $\mathrm{C}-\mathrm{O} / \mathrm{COOH}$ and $\mathrm{C}=\mathrm{O}$ bonds. This also applies for SH-CNT system. On the other hand, in MO-CNT and HOCNT systems, after $\mathrm{Cu}$ deposition, the concentration of $\mathrm{C}-\mathrm{O} / \mathrm{COOH}$ bonds decreases significantly (Figure S1). This can be due to the fact that in MO-CNT and HO-CNT samples, $\mathrm{Cu}$ deposits are mostly interacting with $\mathrm{C}-\mathrm{O} / \mathrm{C}-\mathrm{OOH}$, whereas in SH-CNT and N-CNT systems the $\mathrm{N}$ and thiol groups bond with $\mathrm{Cu}$. It is worth noting that the $\mathrm{N} 1 \mathrm{~s}$ peak analysis can give a better understanding of the interactions between $\mathrm{Cu}$ and N-CNT. However, this peak was not detected 
after $\mathrm{Cu}$ deposition because of the $\mathrm{N}$ low weight ratio and being covered by large $\mathrm{Cu}$ spheres. On the other hand, the $\mathrm{Cu} 2 \mathrm{p}_{3 / 2}$ peak for other samples similarly show a shift towards a higher binding energy of around $933.5 \mathrm{eV}$. Since each sample has a different fraction of copper oxides, it is deduced that this shift has a different source in each sample. The shift for MO-CNT/Cu is attributed to comparatively higher $\mathrm{CuO}$ content while in $\mathrm{SH}-\mathrm{CNT} / \mathrm{Cu}$ sample, the shift is mostly caused by strong interactions between copper and sulfur of thiol functional group. For further evidence, the S2p $3 / 2$ peak at binding energy of around $164 \mathrm{eV}$ needs to be studied. However, this peak disappeared in the SH-CNT hybrid system due to its low content and being covered by the metallic particles.

To confirm the crystalline properties of the nanocrystals, the as-synthesized hybrid systems were examined using XRD. As presented in Figure 5, the nanoparticles display high crystallinity. Due to low content, $\mathrm{Sn}$ and Pd peaks are not detected. The detected peaks belong mostly to a lower oxidized state of $\mathrm{Cu}$, i.e., $\mathrm{Cu}_{2} \mathrm{O}$ and metallic $\mathrm{Cu}$. In carboxyl-treated systems, i.e., MO-CNT/Cu and $\mathrm{HO}-\mathrm{CNT} / \mathrm{Cu}$, diffraction peaks at $2 \theta$ of $36.5^{\circ}, 42.5^{\circ}, 61.6^{\circ}$, and $73.4^{\circ}$ are present which can be indexed as the (111), (200), (220), and (311) planes of $\mathrm{Cu}_{2} \mathrm{O}$, respectively (JCPDS card no. 01-080-3714). In addition, the XRD patterns of N-CNT/Cu and SH-CNT/Cu systems show peaks at (200) peaks at $43.2^{\circ}, 50.4^{\circ}$ and $74.1^{\circ}$ which can be identified as (111), (200), and (220) planes of metallic copper (JCPDS card no. 01-071-3761). Due to relatively low content of CNT, only a weak peak of (200) graphite is detected at $2 \theta$ of $26^{\circ}$ (JCPDS card no. 01-071-3739). Differentiation of (111) and (220) peaks of $\mathrm{Cu}$ from (200) and (311) peaks of $\mathrm{Cu}_{2} \mathrm{O}$ is challenging since they are situated very close to each other (compare $43.2^{\circ}$ and $74.1^{\circ}$ for $\mathrm{Cu}$ to $42.5^{\circ}$ and $73.4^{\circ}$ for $\mathrm{Cu}_{2} \mathrm{O}$ ). Here, these peaks are located at slightly lower degrees for carboxylactivated samples. Therefore, they are attributed to $\mathrm{Cu}_{2} \mathrm{O}$. Also, the metallic copper (200) peak 
at $50.4^{\circ}$ only appears for $\mathrm{SH}-\mathrm{CNT} / \mathrm{Cu}$ and $\mathrm{N}-\mathrm{CNT} / \mathrm{Cu}$ samples. As shown, the strong $\mathrm{Cu}_{2} \mathrm{O}$ peak at $2 \theta$ of $36.5^{\circ}$ is common in all samples along with a weak (220) peak of $\mathrm{Cu}_{2} \mathrm{O}$ at $61.6^{\circ}$. However, the intensity of these common peaks is relatively lower in amine and thiol treated samples. These results confirm the XPS analyses, indicating that $\mathrm{Cu}$ is present mainly in oxide state in $\mathrm{MO}-\mathrm{CNT} / \mathrm{Cu}$ and $\mathrm{HO}-\mathrm{CNT} / \mathrm{Cu}$ samples while there is metallic copper along with $\mathrm{Cu}_{2} \mathrm{O}$ in $\mathrm{SH}-\mathrm{CNT} / \mathrm{Cu}$ and $\mathrm{N}-\mathrm{CNT} / \mathrm{Cu}$. The presence of metallic copper in amine-treated sample is expected since most of the reduced copper are large particles formed in the solution which are not as easily oxidized. In other words, in amine-treated sample, unlike the thiol-activated one, formation of metallic copper is not related to the $\mathrm{CNT}$ interface but is a result of larger $\mathrm{Cu}$ particle size and lower surface area. Finally, in all samples, the [111] faces are the dominant crystal orientation regardless of the oxidation state of deposits. This is due to the fact that growth direction strongly depends on parameters such as complexing agent and $\mathrm{pH}$ which is similar in all samples $[39,52]$. It is worth mentioning that the direction of growth for $\mathrm{Cu}$-based nanoparticles becomes important in catalysis applications since some of the planes have higher reactivity $[41]$. 


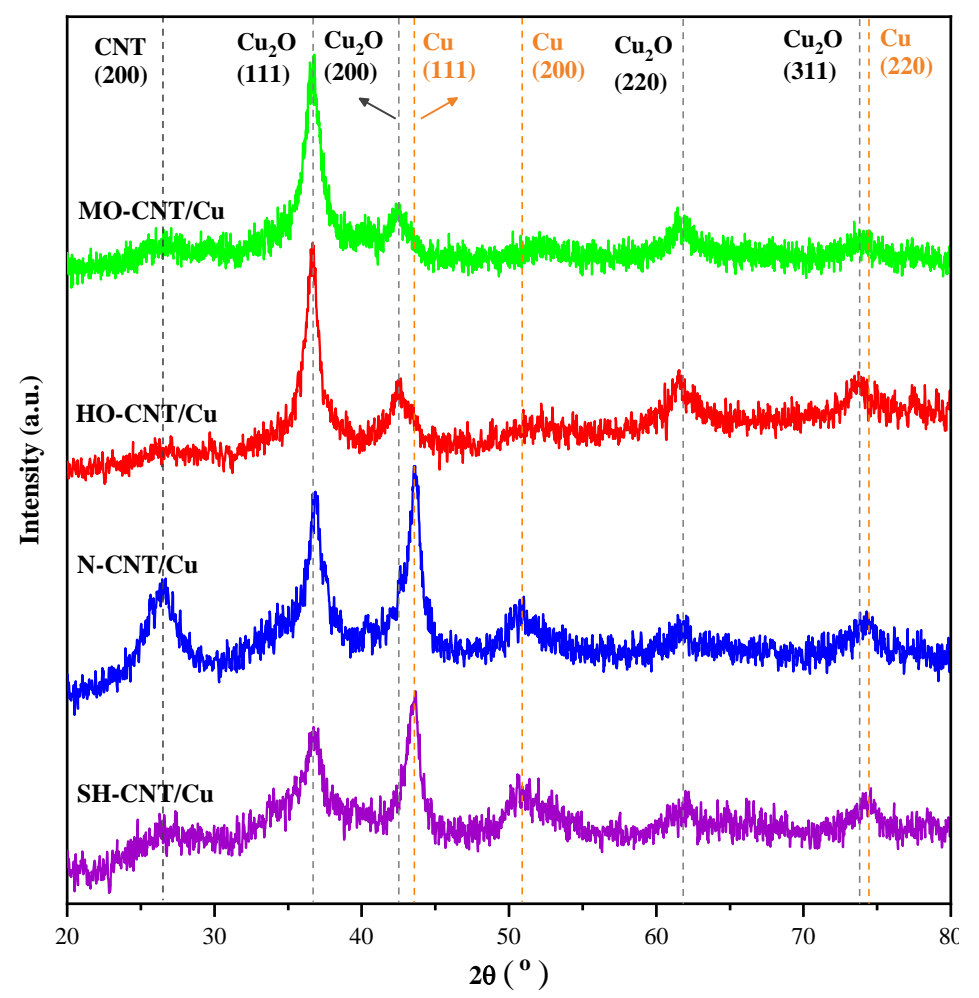

Figure 5. XRD patterns of hybrid systems. These results show that $\mathrm{N}-\mathrm{CNT} / \mathrm{Cu}$ and $\mathrm{SH}-\mathrm{CNT} / \mathrm{Cu}$ systems mostly contain metallic copper while the MO-CNT/CU system contain significant amounts of $\mathrm{CuO}$. Sn or Pd are not detected.

\subsection{Electrical conductivity}

The thin films with different CNT surface functional groups have a similar weight of around 300 mg after $\mathrm{Cu}$ electroless deposition, suggesting they have equal amount of copper. This means each thin film contains around $16 \mathrm{wt} \%$ (45 vol\%) of f-CNTs and the composite thin film has a theoretical density of $5.8 \mathrm{~g} / \mathrm{cm}^{3}$. Consequently, it can be deduced that any difference in the electrical conductivity is the results of the interaction between $\mathrm{Cu}$ and CNTs containing different surface functional group types. The electrical conductivity measurement results are presented in Table 2. The SH-CNT/Cu film has the highest conductivity of $0.82 \mathrm{MS} / \mathrm{m}$, which is more than seven times higher than that of the $\mathrm{N}-\mathrm{CNT} / \mathrm{Cu}$ sample. The conductivity of samples containing carboxyl-activated CNTs, i.e., MO-CNT/Cu and $\mathrm{HO}-\mathrm{CNT} / \mathrm{Cu}$, are similar at around 0.20 MS/m. 
Indeed, the thiol groups show the highest affinity towards copper. TEM results show that all the SH-CNTs are coated and copper is able to effectively wet SH-CNT surfaces. Also, based on XPS results, copper deposits have the strongest interaction with SH-CNT and are better preserved against oxidation. As a result, these thiol functionalized sites act as preferred sites for deposition of copper and promote uniform deposition of copper (Figure S6). This leads to higher conductivity of SH-CNT/Cu system compared with other systems. Compared with MO-CNTs, HO-CNTs show better affinity towards $\mathrm{Cu}$. However, their composite film shows a slightly lower conductivity. XPS results show that, as a result of the harsh acid treatment, the defects population increases significantly. These defects, specifically oxygen in carboxyl groups, can scatter the electrons and impair ballistic conductance of CNTs [53]. More importantly, harsh acid treatment can shorten the length of CNTs which can reduce the conductivity as a result of increase junctions of CNTs and the matrix [54].

The N-CNT/Cu system shows the lowest conductivity. Although it has been reported that Ndoping will cause the least disturbance in the pure $\mathrm{sp}^{2}$ structure of $\mathrm{CNT}$ and can even enhance the conductivity of $\mathrm{Cu} / \mathrm{CNT}$ systems [23], N-CNTs are not seeded effectively and show the lowest affinity towards copper, hence resulting in the lowest electrical conductivity due to hydrophobicity and stereo-hindrance effect. Examining the cross section of the thin films reveals that the $\mathrm{N}-\mathrm{CNT} / \mathrm{Cu}$ thin films were not as compact as other systems and $\mathrm{Cu}$ deposits formed discrete patches (Figure S6 c and g); while for $\mathrm{SH}-\mathrm{CNT} / \mathrm{Cu}$ system, $\mathrm{Cu}$ was more uniformly distributed within the thin film (Figure S6 e and f).

The above findings show that functional groups on the surface of CNTs have a great effect on the morphology, composition and final properties of CNT-Cu composites. It should be noted that length and quality of CNTs are very important factors which can significantly affect the final 
properties of the composite. In this work we used commercially available CNTs but it is speculated that, by using longer CNTs, higher conductivities can be achieved.

By understanding the effect of CNT surface functionalization on the deposited $\mathrm{Cu}$ morphology and composition, we can design new hybrid systems for specific application needs. For example, thiol activation can remarkably enhance the wettability of CNT surface towards $\mathrm{Cu}$ and improve the electrical conductivity of $\mathrm{CNT} / \mathrm{Cu}$ system. As a result, portable and flexible electronics, and nanocomposites for structural applications can be benefitted from the uniform coating of $\mathrm{Cu}$ on SH-CNTs. On the other hand, in application where surface area and reactivity are important, such as catalysis and energy storage, carboxylated and N-doped CNTs can perform better. Also, the above findings are not limited to $\mathrm{CNT} / \mathrm{Cu}$ systems and can be applied to other carbon nanostructures, such as graphene and other transitional metals, including $\mathrm{Ni}, \mathrm{Co}$, and $\mathrm{Pd}$.

Table 2. Electrical conductivity and specific conductivity of thin films made of pristine CNTs and different CNT/Cu systems.

\begin{tabular}{lll}
\hline Sample & $\begin{array}{l}\text { Electrical } \\
\text { conductivity }(\mathrm{S} / \mathrm{m})\end{array}$ & $\begin{array}{l}\text { Specific conductivity } \\
\left(\mathrm{Sm}^{2} / \mathrm{Kg}\right)^{*}\end{array}$ \\
\hline P-CNTs & $3 \times 10^{3}( \pm 760)$ & 0.33 \\
$\mathrm{MO}-\mathrm{CNT} / \mathrm{Cu}$ & $1.9 \times 10^{5}( \pm 2000)$ & 32.76 \\
$\mathrm{HO}-\mathrm{CNT} / \mathrm{Cu}$ & $2.0 \times 10^{5}( \pm 2100)$ & 34.48 \\
$\mathrm{~N}-\mathrm{CNT} / \mathrm{Cu}$ & $9.8 \times 10^{4}( \pm 1860)$ & 16.90 \\
$\mathrm{SH}-\mathrm{CNT} / \mathrm{Cu}$ & $8.2 \times 10^{5}( \pm 1530)$ & 141.38 \\
\hline
\end{tabular}

* Specific conductivity numbers are based on theoretical densities.

\section{Conclusion}

CNTs were functionalized by N-doping, carboxyl (7 at\% and 12 at $\%$, respectively) and thiol groups. They were subsequently coated with copper via electroless deposition approach. The interaction between CNT functionalized with the above four different surface functional groups and copper were examined. By utilizing HRTEM, STEM, EDX, XPS, and XRD, the effect of 
CNT surface functional groups on the interaction, morphology and composition of copper deposits were studied in detail. The nanoscale analyses have revealed how the CNTs surface functional groups interact with $\mathrm{Cu}$ to form different morphologies and chemical states. Moreover, the effect of CNT surface functionality on the electrical conductivity of CNT/Cu films produced by vacuum filtration was analyzed by using four-point electrical conductivity measurements, indicating that the thiol group can enhance the wettability of the CNTs towards copper deposition. Thiol has lone pairs of electrons and can be deprotonated, resulting in formation of a strong bond with palladium and subsequently copper. Uniformly adsorbed palladium particles on CNT acts as nucleation sites for copper. These nuclei that are located in a proximity of each other, resulting in a dense and uniform coating on SH-CNT surface. This dense coating helps protect against oxidation. On the other hand, the carboxyl treated CNTs do not have the same affinity towards metal ions. The wettability is low and copper will form as discrete and small spheres on the MO-CNT and HO-CNT surfaces which are prone to oxidation due to their higher surface energy state. Moreover, on N-CNTs, spherical Cu particles with diameter around $20 \mathrm{~nm}$ are formed that have minimal contact with the CNT surface. Thus, copper coating on N-CNTs cannot be effectively achieved mainly due to their hydrophobicity and low wettability. Accordingly, thin films made of SH-CNT/Cu system in which copper is seeded uniformly shows the highest electrical conductivity, which is four times higher than composites containing carboxylated CNTs and seven times higher than N-doped CNTs.

\section{Acknowledgments}

All the authors acknowledge the financial support of the Lloyd's Register Foundation, London, UK, who has funded this research through their grants to protect life and property by supporting engineering-related education, public engagement and the application of research. 


\section{References}

1. Eder, D., Carbon nanotube- inorganic hybrids. Chemical reviews, 2010. 110(3): p. 1348-1385.

2. Poncharal, P., et al., Room temperature ballistic conduction in carbon nanotubes. 2002, ACS Publications.

3. Behabtu, N., et al., Strong, light, multifunctional fibers of carbon nanotubes with ultrahigh conductivity. science, 2013. 339(6116): p. 182-186.

4. Lekawa-Raus, A., et al., Electrical properties of carbon nanotube based fibers and their future use in electrical wiring. Advanced Functional Materials, 2014. 24(24): p. 3661-3682.

5. Zhang, S., et al., Carbon-Nanotube-Based Electrical Conductors: Fabrication, Optimization, and Applications. Advanced Electronic Materials, 2019: p. 1800811.

6. Zhang, S., et al., Ultra-high conductivity and metallic conduction mechanism of scale-up continuous carbon nanotube sheets by mechanical stretching and stable chemical doping. Carbon, 2017. 125: p. 649-658.

7. Hjortstam, O., et al., Can we achieve ultra-low resistivity in carbon nanotube-based metal composites? Applied Physics A, 2004. 78(8): p. 1175-1179.

8. Subramaniam, C., et al., One hundred fold increase in current carrying capacity in a carbon nanotube-copper composite. Nature communications, 2013. 4: p. 2202.

9. Kim, J.A., et al., Effects of surface modification on rheological and mechanical properties of CNT/epoxy composites. Carbon, 2006. 44(10): p. 1898-1905.

10. Ma, P.-C., et al., Dispersion and functionalization of carbon nanotubes for polymer-based nanocomposites: a review. Composites Part A: Applied Science and Manufacturing, 2010. 41(10): p. 1345-1367.

11. Yang, S.-Y., et al., Effect of functionalized carbon nanotubes on the thermal conductivity of epoxy composites. Carbon, 2010. 48(3): p. 592-603.

12. Sahoo, N.G., et al., Polymer nanocomposites based on functionalized carbon nanotubes. Progress in polymer science, 2010. 35(7): p. 837-867.

13. Daneshvar-Fatah, F. and F. Nasirpouri, A study on electrodeposition of Ni-noncovalnetly treated carbon nanotubes nanocomposite coatings with desirable mechanical and anti-corrosion properties. Surface and Coatings Technology, 2014. 248: p. 63-73.

14. Nasirpouri, F., et al., Magnetic Properties of Electrodeposited Nickel-Multiwall Carbon Nanotube Composite Films. IEEE Transactions on Magnetics, 2015. 51(11): p. 1-4.

15. Cha, S.I., et al., Extraordinary strengthening effect of carbon nanotubes in metal-matrix nanocomposites processed by molecular-level mixing. Advanced Materials, 2005. 17(11): p. 13771381.

16. Wildgoose, G.G., C.E. Banks, and R.G. Compton, Metal nanoparticles and related materials supported on carbon nanotubes: methods and applications. Small, 2006. 2(2): p. 182-193.

17. Bakshi, S.R., D. Lahiri, and A. Agarwal, Carbon nanotube reinforced metal matrix composites-a review. International Materials Reviews, 2010. 55(1): p. 41-64.

18. Nai, M.H., J. Wei, and M. Gupta, Interface tailoring to enhance mechanical properties of carbon nanotube reinforced magnesium composites. Materials \& Design, 2014. 60: p. 490-495.

19. Daneshvar, F., et al., Porous $\mathrm{SnO}_{2}-\mathrm{Cu}_{x} \mathrm{O}$ nanocomposite thin film on carbon nanotubes as electrodes for high performance supercapacitors. Nanotechnology, 2018. 30(1): p. 015401.

20. Wu, B., et al., Noble metal nanoparticles/carbon nanotubes nanohybrids: synthesis and applications. Nano Today, 2011. 6(1): p. 75-90.

21. Ilari, G.M., et al., Carbon-metal interfaces analyzed by aberration-corrected TEM: How copper and nickel nanoparticles interact with MWCNTs. Micron, 2015. 72: p. 52-58.

22. Xue, Y., et al., Quantifying thiol-gold interactions towards the efficient strength control. Nature communications, 2014. 5: p. 4348. 
23. Milowska, K.Z., et al., Carbon nanotube functionalization as a route to enhancing the electrical and mechanical properties of $\mathrm{Cu}-\mathrm{CNT}$ composites. Nanoscale, 2019. 11(1): p. 145-157.

24. Oluwalowo, A., et al., Electrical and thermal conductivity improvement of carbon nanotube and silver composites. Carbon, 2019. 146: p. 224-231.

25. Singh, B., et al., Pt based nanocomposites (mono/bi/tri-metallic) decorated using different carbon supports for methanol electro-oxidation in acidic and basic media. Nanoscale, 2011. 3(8): p. 33343349.

26. Sharifi, T., et al., Nitrogen doped multi walled carbon nanotubes produced by CVD-correlating XPS and Raman spectroscopy for the study of nitrogen inclusion. Carbon, 2012. 50(10): p. 3535-3541.

27. Van Tam, T., S.H. Hong, and W.M. Choi, Facile synthesis of cysteine-functionalized graphene quantum dots for a fluorescence probe for mercury ions. RSC Advances, 2015. 5(118): p. 9759897603.

28. Bosch-Navarro, C., J.P. Rourke, and N.R. Wilson, Controlled electrochemical and electroless deposition of noble metal nanoparticles on graphene. RSC Advances, 2016. 6(77): p. 73790-73796.

29. Banhart, F., Interactions between metals and carbon nanotubes: at the interface between old and new materials. Nanoscale, 2009. 1(2): p. 201-213.

30. Datsyuk, V., et al., Chemical oxidation of multiwalled carbon nanotubes. Carbon, 2008. 46(6): p. 833-840.

31. Czerw, R., et al., Identification of electron donor states in $N$-doped carbon nanotubes. Nano Letters, 2001. 1(9): p. 457-460.

32. Showkat, A.M., et al., Dispersion of gold nanoparticles into thiol-functionalized carbon nanotubes by $\gamma$-radiation. Diamond and related materials, 2007. 16(8): p. 1688-1692.

33. Kim, Y.-T. and T. Mitani, Surface thiolation of carbon nanotubes as supports: A promising route for the high dispersion of Pt nanoparticles for electrocatalysts. Journal of catalysis, 2006. 238(2): p. 394401.

34. Pakiari, A. and Z. Jamshidi, Nature and strength of $M-S$ Bonds ( $M=A u, A g$, and $C u$ ) in binary alloy gold clusters. The Journal of Physical Chemistry A, 2010. 114(34): p. 9212-9221.

35. Wan, Y., et al., Electrochemical formation and reduction of copper oxide nanostructures in alkaline media. Electrochemistry Communications, 2013. 36: p. 99-102.

36. Biesinger, M.C., et al., Resolving surface chemical states in XPS analysis of first row transition metals, oxides and hydroxides: Sc, Ti, V, Cu and Zn. Applied Surface Science, 2010. 257(3): p. 887898.

37. Poulston, S., et al., Surface oxidation and reduction of $\mathrm{CuO}$ and $\mathrm{Cu} 2 \mathrm{O}$ studied using XPS and XAES. Surface and Interface Analysis: An International Journal devoted to the development and application of techniques for the analysis of surfaces, interfaces and thin films, 1996. 24(12): p. 811-820.

38. Svintsitskiy, D.A., et al., In situ XRD, XPS, TEM, and TPR study of highly active in CO oxidation CuO nanopowders. The Journal of Physical Chemistry C, 2013. 117(28): p. 14588-14599.

39. Mott, D., et al., Synthesis of size-controlled and shaped copper nanoparticles. Langmuir, 2007. 23(10): p. 5740-5745.

40. Kawasaki, H., et al., Microwave-assisted polyol synthesis of copper nanocrystals without using additional protective agents. Chemical Communications, 2011. 47(27): p. 7740-7742.

41. Gawande, M.B., et al., $\mathrm{Cu}$ and $\mathrm{Cu}$-based nanoparticles: synthesis and applications in catalysis. Chemical reviews, 2016. 116(6): p. 3722-3811.

42. Fan, Y., B.R. Goldsmith, and P.G. Collins, Identifying and counting point defects in carbon nanotubes. Nature materials, 2005. 4(12): p. 906.

43. Nasirpouri, F., Electrodeposition of nanostructured materials. 2017: Springer, p. 90-91.

44. Saikova, S., et al., Conditions for the formation of copper nanoparticles by reduction of copper (II) ions with hydrazine hydrate solutions. Russian Journal of General Chemistry, 2010. 80(6): p. 11221127.

45. Lin, Y.-M. and S.-C. Yen, Effects of additives and chelating agents on electroless copper plating. Applied surface science, 2001. 178(1-4): p. 116-126. 
46. Kim, J.Y., et al., Preparation, characterization and catalytic properties of Pd-decorated carbon nanotubes possessing different linkers. Journal of Materials Chemistry, 2011. 21(16): p. 5999-6005.

47. Zhang, C., et al., Efficient removal of heavy metal ions by thiol-functionalized superparamagnetic carbon nanotubes. Chemical Engineering Journal, 2012. 210: p. 45-52.

48. Rigo, A., et al., Interaction of copper with cysteine: stability of cuprous complexes and catalytic role of cupric ions in anaerobic thiol oxidation. Journal of inorganic biochemistry, 2004. 98(9): p. 14951501.

49. Ghelichkhah, Z., et al., L-cysteine/polydopamine nanoparticle-coatings for copper corrosion protection. Corrosion Science, 2015. 91: p. 129-139.

50. Kang, J.S. and T.A. Taton, Oligothiol graft-copolymer coatings stabilize gold nanoparticles against harsh experimental conditions. Langmuir, 2012. 28(49): p. 16751-16760.

51. Zhang, H. and H. Cui, Synthesis and characterization of functionalized ionic liquid-stabilized metal (gold and platinum) nanoparticles and metal nanoparticle/carbon nanotube hybrids. Langmuir, 2009. 25(5): p. 2604-2612.

52. Susman, M.D., et al., Chemical deposition of $\mathrm{Cu}_{2} \mathrm{O}$ nanocrystals with precise morphology control. ACS nano, 2014. 8(1): p. 162-174.

53. Bockrath, M., et al., Resonant electron scattering by defects in single-walled carbon nanotubes. Science, 2001. 291(5502): p. 283-285.

54. Yan, K., et al., The interface effect of the effective electrical conductivity of carbon nanotube composites. Nanotechnology, 2007. 18(25): p. 255705. 